\title{
Plastic Optical Fiber Sensing of Alcohol Concentration in Liquors
}

\author{
Masayuki Morisawa and Shinzo Muto \\ Graduate School of Medicine and Engineering, University of Yamanashi 4-3-11 Takeda, Kofu, 400-8511, Japan \\ Correspondence should be addressed to Masayuki Morisawa, morisawa@yamanashi.ac.jp
}

Received 1 December 2011; Revised 30 January 2012; Accepted 2 February 2012

Academic Editor: Wolfgang Ecke

Copyright ( $) 2012$ M. Morisawa and S. Muto. This is an open access article distributed under the Creative Commons Attribution License, which permits unrestricted use, distribution, and reproduction in any medium, provided the original work is properly cited.

\begin{abstract}
A simple optical fiber sensing system of alcohol concentration in liquors has been studied. In this sensor head, a mixture polymer of novolac resin and polyvinylidenefluoride (PVDF) with a ratio of 9:1 was coated as a sensitive cladding layer on the plastic fiber core made of polystyrene-(PS-)coated polycarbonate (PC). Using this sensor head and a green LED light source, it was confirmed that alcohol concentration in several kinds of liquors from beer to whisky can easily be measured with a fast response time less than 1 minute.
\end{abstract}

\section{Introduction}

In every-day life, brewers supply several liquors (alcoholic drinks) such as beer, brandy, wine, and whisky, and people are fond of them. However, in brewery, it takes many times to check the alcohol concentration in product liquors, for example, using a distillation method [1] and gas or liquid chromatography [2]. Therefore, development of a simple and quick detection system of alcohol concentration in liquors is strongly required.

From the above view point, we have studied an optical measurement system of alcohol concentration in liquors using a plastic optical fiber (POF) because the POF has many advantages such as low cost, easiness in handling, and immunity from electrical interface [3-6]. This paper reports on its configuration and basic sensing properties of alcohol concentration in some alcoholic drinks.

\section{Sensor Head Structure and Its Sensing Principle}

A certain kind of polymers such as novolac swells when it is exposed to vapor-phase alcohol [7] or alcohol solutions. This effect produces a change in refractive index of that film. Figure 1 shows a change in the refractive index of novolac resin when exposed to ethanol, determined using an Abbe refractometer. As can be seen from this figure, after being exposed to the ethanol $(\mathrm{EtOH})$, the refractive index in the novolac film decreased remarkably from $n_{D}=1.61$ to 1.46 . In opposition, when is ethanol exhausted from the film, its refractive index returned to the initial value. This reversible property is more suitable to construct an optical sensor.

Using this property in the novolac film, we fabricated the POF-type sensor head. Figure 2 shows schematics of the sensor head structure and operation models with and without alcohol, respectively. In this POF sensor, the marketed polycarbonate (PC) fiber with a refractive index of $n_{D}=1.58$ was used after removing the cladding layer. The polystyrene (PS) layer with a refractive index of $n_{D}=1.60$ was coated on the PC fiber surface because the PC surface was broken by the solvent for novolac solution. Referring to the preliminary experiment [7], the novolac/iron complex (novolac/Fe: SO) was found to be superior to novolac resin only. Therefore, we used it as an alcohol sensitive material.

In Figure 2(a), the sensor head operates as a leaky POF (i.e., $n_{1}<n_{2}$ ), and the intensity of output light passing through it is very weak. In this case, by using a ray tracing method, the calculated total output light intensity $P_{\text {outl }}$ is given by

$$
P_{\text {out } 1}=\sum_{\theta=0}^{\theta_{c}} P(\theta) r(\theta)^{m},
$$




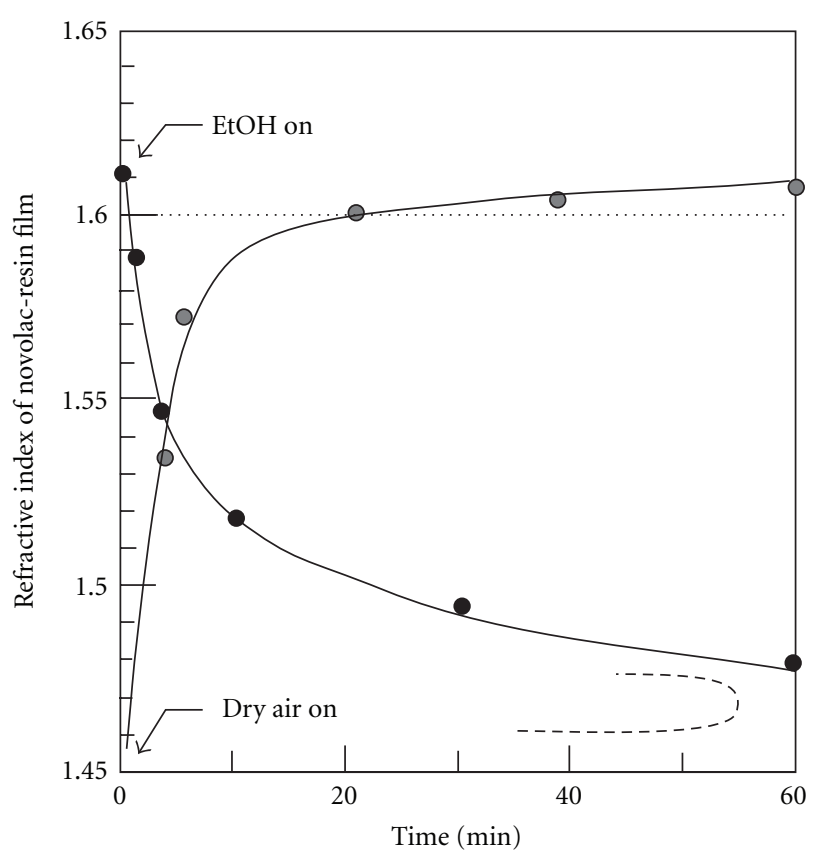

FIgURE 1: Refractive index change in novolac film with thickness of about $5 \mu \mathrm{m}$.

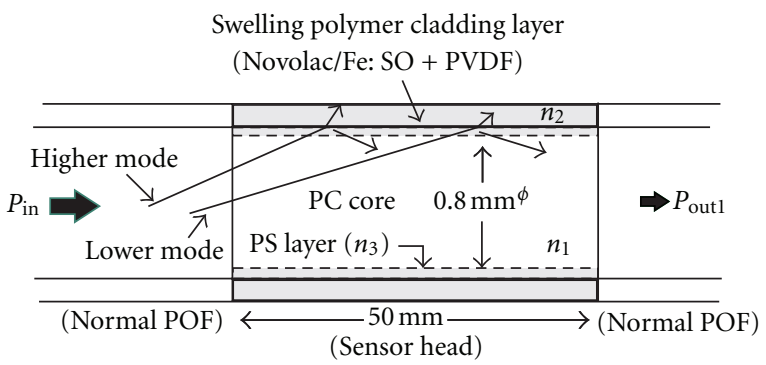

(a) Without alcohol: $n_{2}>n_{1}$ (leaky-POF structure)

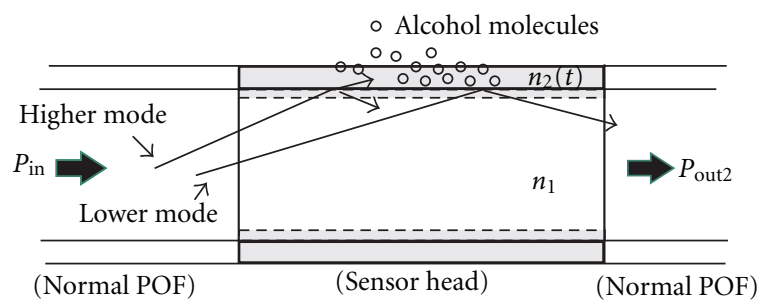

(b) With alcohol: $n_{2}(t)<n_{1}$ (guided-POF structure)

FIgURE 2: Sensor head structure.

where $P(\theta)$ is the optical power for a ray with an angle $\theta, \theta_{c}$ is the critical angle at the input side of the POF, $r(\theta)$ is the power reflection coefficient, and $m$ is the reflection number in the sensor head.

On the other hand, when exposed to alcohol, the cladding layer swells, and its refractive index begins to decrease, becoming lower than that of the fiber core (i.e., $n_{1}>$ $n_{2}$ ). Then, as shown in Figure 2 (b), the sensor head changes to a guided POF, and the output light intensity increases

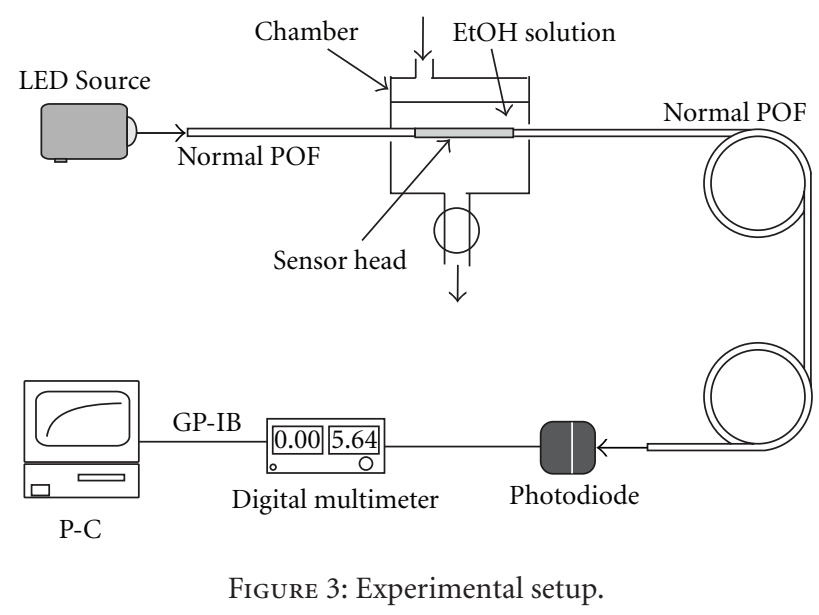

remarkably. In this case, the total output light intensity $P_{\text {out2 }}$ of the sensor head is given by

$$
P_{\text {out } 2}=\sum_{\theta=0}^{\theta_{c s}} P(\theta)+\sum_{\theta=\theta_{c s}}^{\theta_{c}} P(\theta) r^{m},
$$

where $\theta_{c s}$ is the critical angle of the sensor head. The value of $P_{\text {out } 2} / P_{\text {out } 1}$ describes the relative change in the output light intensity depending on the alcohol.

As is evident from (1) and (2), for enabling this sensor to respond to alcohol at a low concentration, the difference between the initial refractive index of the cladding and that of core is required to be decreased so that switching from the leaky to the waveguide modes becomes easy. Hence, we have mixed low-refractive-index polyvinylidenefluoride (PVDF; $n_{D}=1.42$ ) with novolac resin so that the refractive index of the cladding polymer approaches that of the PC (polycarbonate) core. The mixed film of novolac/Fe:SO and PVDF with a ratio of 3 to 1 was coated on the above PScoated-PC fiber (PS/PC) with a core diameter of $0.8 \mathrm{~mm}$, by means of a dip coating.

Next, we consider the effect of the intermediate protective PS layer. In terms of a ray-tracing technique based on the Snell's law

$$
n_{1} \sin \theta_{1}=n_{3} \sin \theta_{3}=n_{2} \sin \theta_{2},
$$

where $n_{3}$ is the refractive index of PS. If the refractive index of the PC core layer, $n_{1}$, is smaller than that of the intermediate PS layer, $n_{3}$ (i.e., $n_{1}<n_{3}$ ), the total reflection of light from the core at the cladding interface (on the core side) is determined from the refractive index of the PC core layer, $n_{1}$, and that of the cladding layer, $n_{2}$, regardless of the presence or absence of the intermediate PS layer. Therefore, the intermediate PS layer does not affect the total reflection.

\section{Experimental Setup}

Figure 3 shows the experimental setup. The POF-type sensor head was set in the small chamber and was connected to the normal POFs on its input and output side. A green LED 


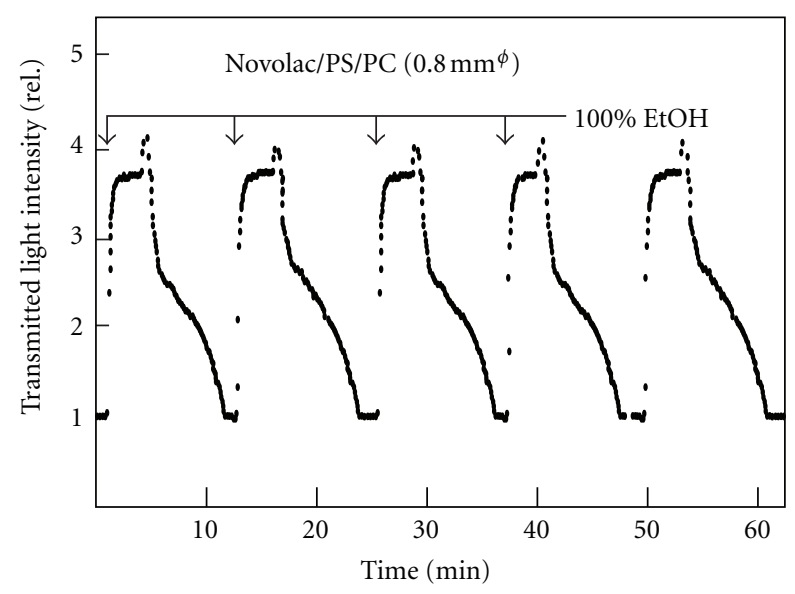

FIgURE 4: Sensor response to $100 \%$ ethanol solution.

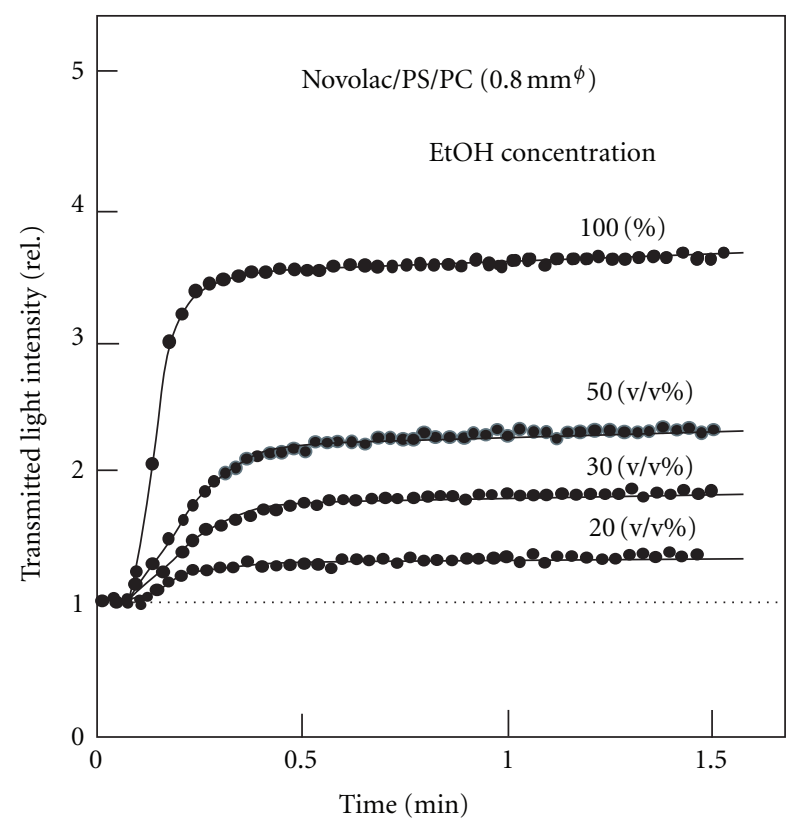

FIGURE 5: Output light intensity change for various concentrations of ethanol.

light with a wavelength of about $530 \mathrm{~nm}$ was guided through the input-side POF, and the transmitted light intensity was measured at the end of output-side POF using a photodiode. The output electrical signal was fed to a digital voltmeter and a computer for data processing. As sensing samples, ethanol solutions diluted by water and several kinds of marketed liquor were used.

\section{Experimental Results}

First, the sensor response for $100 \%$ ethanol was checked. Its result is shown in Figure 4. From this figure, it is found that the transmitted light intensity passing through the sensor head (output light intensity) increased remarkably as soon as ethanol was injected and then reached a stable value after about $1 \mathrm{~min}$. In addition, this system showed
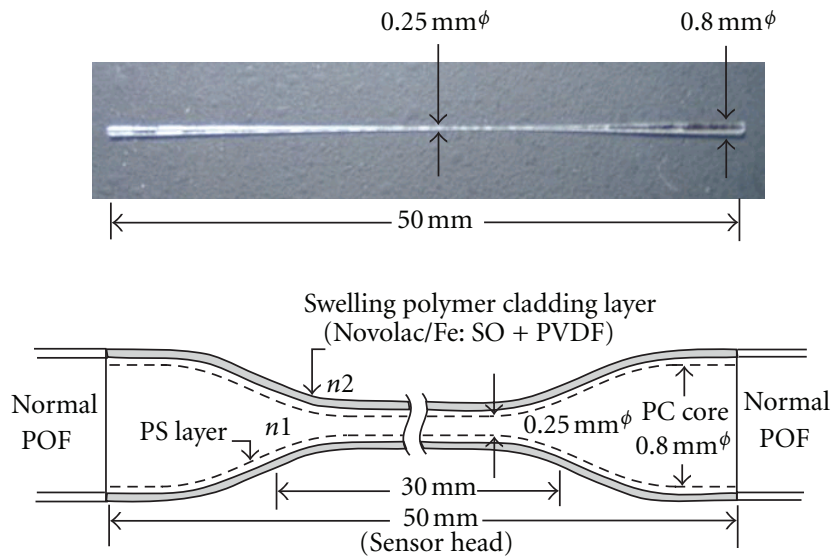

Figure 6: Photograph and illustrated structure of taper-type POF sensor head.

good reproducibility. On the other hand, when the ethanol solution was ejected, the spiky signal suddenly appeared and then the output intensity began to decrease slowly. These phenomena are due to residual alcohol droplets attached on the sensor head surface. In fact, after sending dry air to the sensor head by using a blower, the output intensity returned to the initial value. So, next, the change in output light intensity against various ethanol solutions was measured. The result is shown in Figure 5.

From this figure, it is found that the output light intensity increases with fast response time less than $0.5 \mathrm{~min}$. Thus, the presented POF sensor operated as a quick and good ethanol-concentration sensor over the range of about $20 \mathrm{v} / \mathrm{v} \%$ to $100 \mathrm{v} / \mathrm{v} \%$. However, it did not operate for the low-ethanol-concentration region below $10 \mathrm{v} / \mathrm{v} \%$. So, further improvement was needed to obtain higher sensitivity.

\section{Fabrication of Taper-Type POF Sensor Head}

As shown in Figure 2, lower modes in this POF-type sensor head mainly contribute to the sensitivity. Therefore, to improve sensitivity, it is better to use a sensor head with a small core diameter. So we tried to fabricate a taper-type POF sensor head by means of a solution etching. The PC fiber with a core diameter of $0.8 \mathrm{~mm}$ and a length of $50 \mathrm{~mm}$ was etched by immersing in 1,4-dioxane for about 1 hour at room temperature and then the PS layer and the novolac/Fe:SO + PVDF sensitive cladding layer were dip-coated on it in turn, as shown in the illustrated sensor head structure in Figure 6.

\section{Measurement of Alcohol Concentration in Liquors}

The change in output light intensity against a various ethanol concentration was measured using the new tapertype POF sensor head. Its result is shown in Figure 7. As is clear from this figure, it became possible to measure a low concentration of ethanol solution below $5 \mathrm{v} / \mathrm{v} \%$. In addition, if we compare these data with the results obtained using 


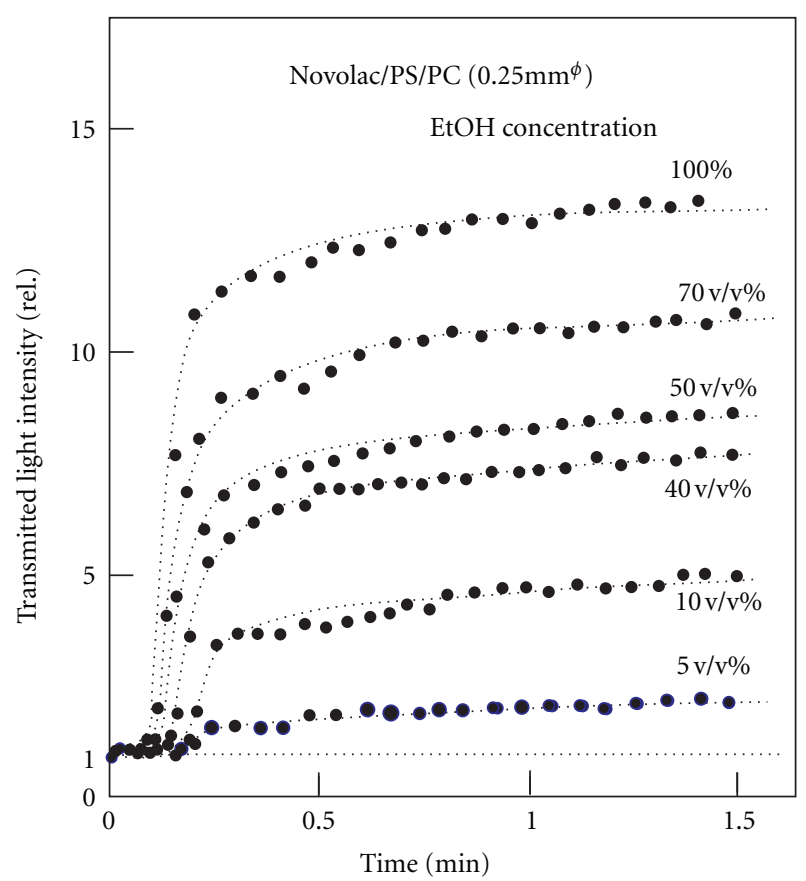

Figure 7: Response of the taper-type sensor head for various concentrations of ethanol.

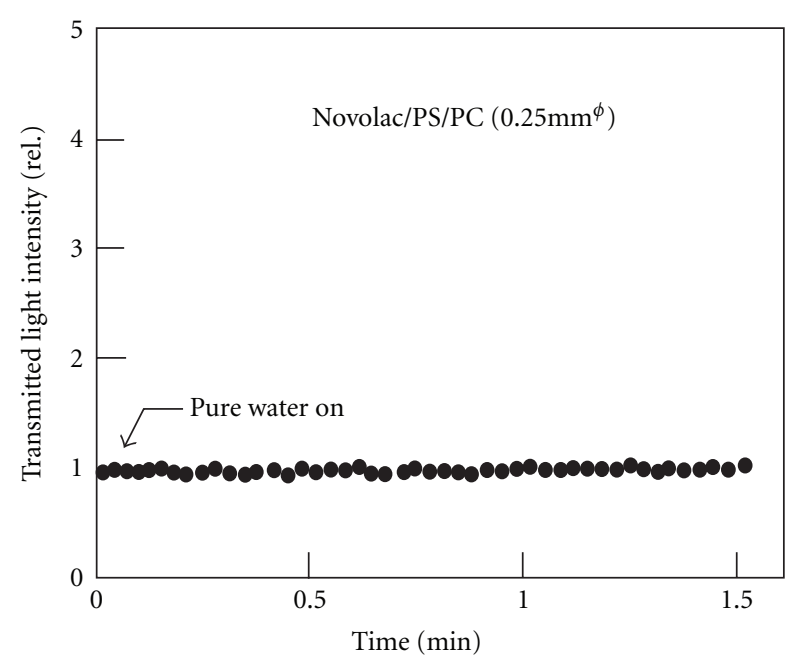

FIGURE 8: Sensor response to water only.

non tapered sensors as shown in Figure 5, we can observe that the change in the intensity of the transmitted light with the concentration of each component becomes so large that the sensitivity is improved by at least a factor of three. This result shows that this sensor can be used for detecting alcohol concentration of several real liquors.

In addition, the result in Figure 8 shows that this sensor does not receive any influence from water. The change in the light intensity with temperature is shown in Figure 9. The light intensity has a negligible dependence on temperature. These properties are also very suitable for a real application. Through these experiments, a calibration curve

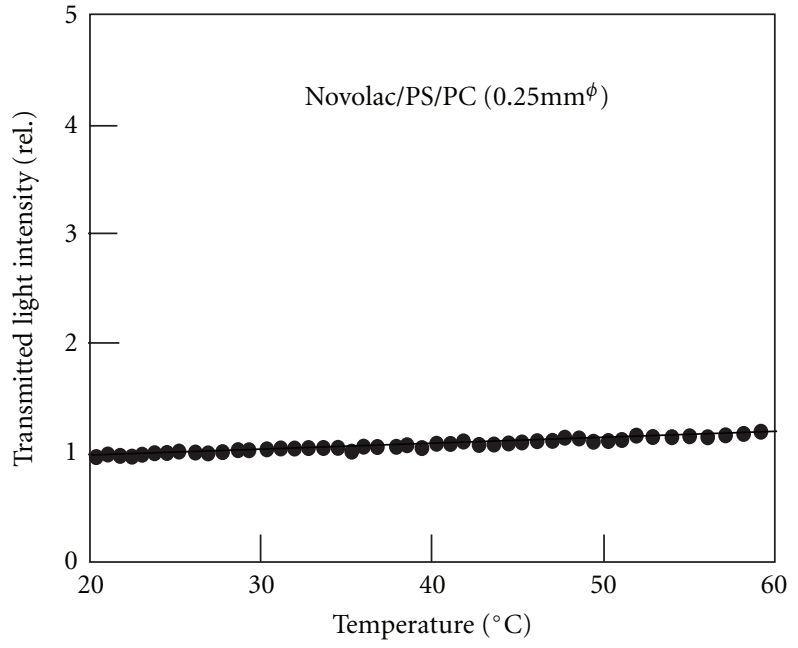

FIgUre 9: The temperature dependence of the POF sensor.

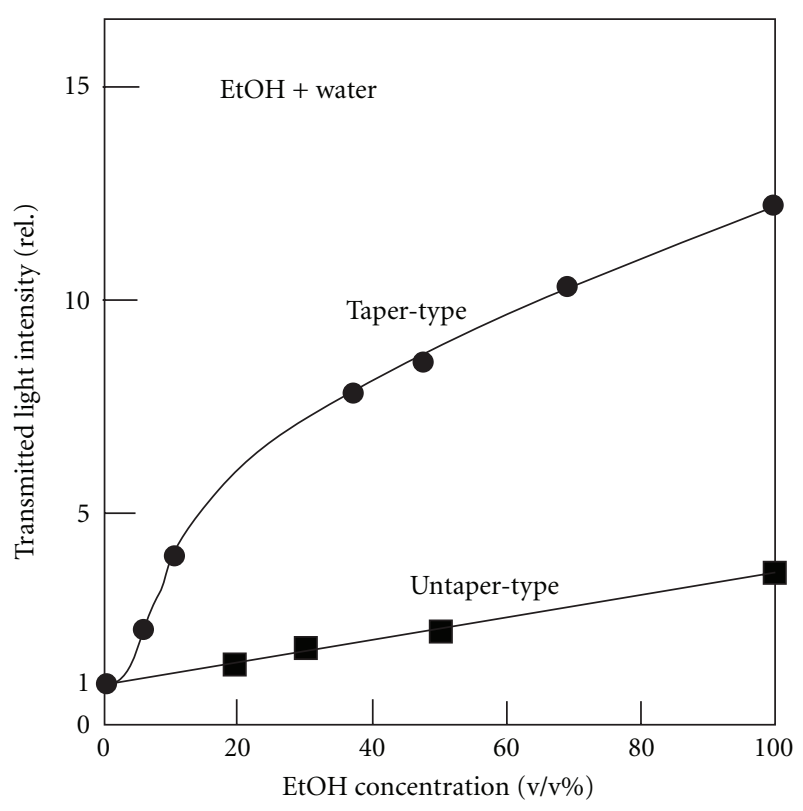

FIGURE 10: Calibration curve against ethanol concentration.

against ethanol concentration could be obtained as shown in Figure 10. In addition, the response does not depend on the concentrations of sugar and salt or acid and alkali, which are potentially contained in alcoholic beverages. This is because novolac resin in the cladding polymer does not exhibit swelling due to these components.

So, next, we tried to measure the alcohol concentration in real liquors such as beer, "Ume" brandy, red wine, white wine, and whisky, which were on the market. The result is shown in Figure 11. The data for white wine is not shown in Figure 11 because it almost overlapped the one of red wine. As can be seen from this figure, alcohol concentration in real liquors can be measured without receiving any influence of color substance and bubble in liquors. 


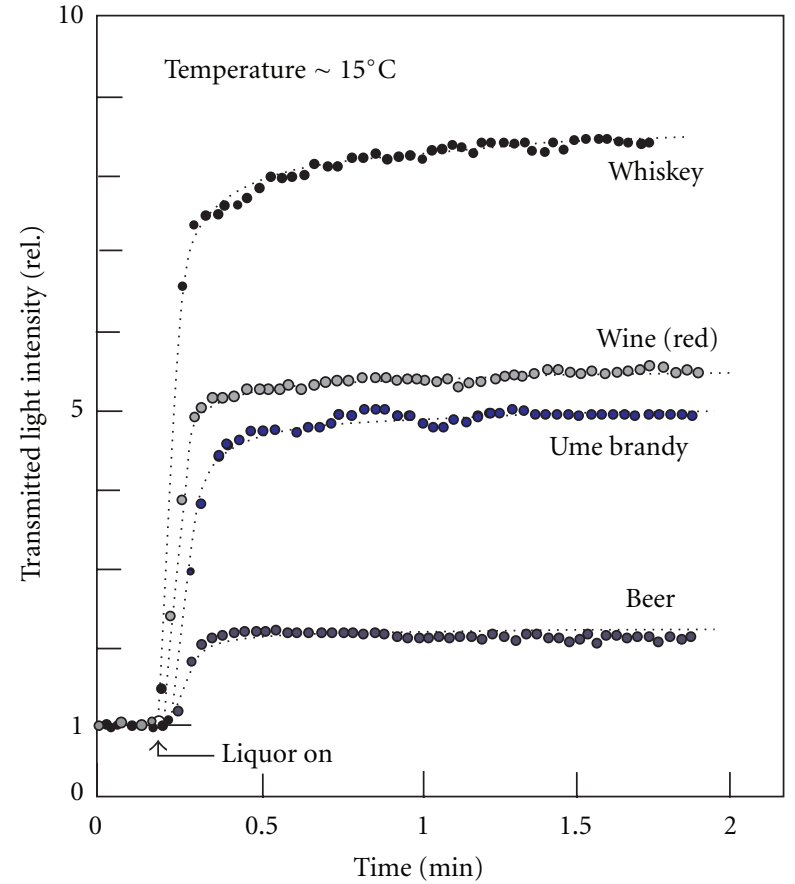

FIGURE 11: Transmitted light intensity change for several kinds of marketed liquor.

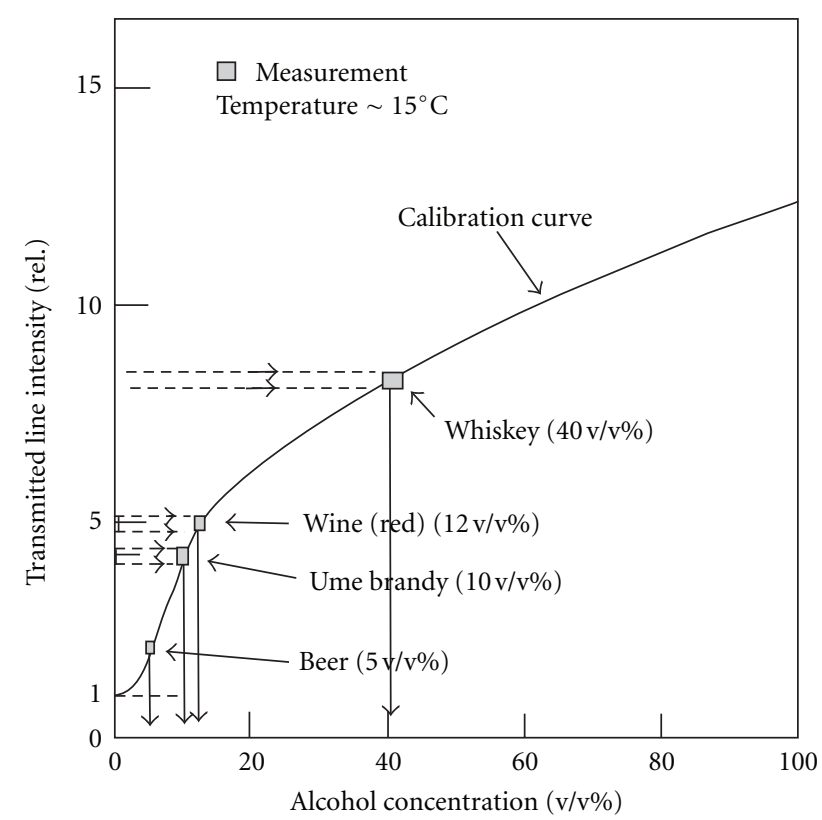

FIgURE 12: Measured alcohol concentration properties. The value of alcohol concentration on the label of liquor bottles are also shown in brackets.

Using the value of the output light intensity at about 1 minute after and the obtained calibration curve in Figure 10, the main result in this paper could easily be obtained as shown in Figure 12. This figure shows that the measured alcohol concentration fitted well to the one described on the label of real liquor bottle with an accuracy of $\pm 0.5 \mathrm{v} / \mathrm{v} \%$.
Namely, usefulness of the presented POF-type alcohol concentration sensor was demonstrated.

\section{Conclusions}

A novel and very simple POF-type sensor for detecting alcohol concentration in liquors, which is based on the POF structure change in sensor head from leaky-type to guidedone, has been studied experimentally. Using this sensor head with a small core diameter of about $0.25 \mathrm{~mm}$, optical detection of the alcohol concentration in real liquors, for example, from beer $(5 \mathrm{v} / \mathrm{v} \%)$ to whisky $(40 \mathrm{v} / \mathrm{v} \%)$ could easily be obtained with a fast response time less than $1 \mathrm{~min}$. Therefore, this sensor will be available for use in any brewery. In addition, the sensitive cladding polymer used here also responds to methanol in vapor phase and solution. Therefore, the presented POF-type alcohol sensor may be used for monitoring leakage of methanol in methanol battery system. Such a study is underway in our laboratory and its result will be reported in the near future.

\section{References}

[1] L. Nykanen and H. Suomalainen, Aroma of Beer, Wine and Distilled Alcoholic Beverages, Akademie, Berlin, Germany, 1983.

[2] E. Peynaud, Knowing and Making Wine, John Wiley \& Sons, New York, NY, USA, 1984.

[3] A. Weinert, Plastic Optical Fibers, Publics MCD, Munich, Germany, 1999.

[4] POF Consortium in Japan, Ed., Plastic Optical Fiber, Kyoritsu, Tokyo, Japan, 1997.

[5] J. Dakin and B. Culshaw, Optical Fiber Sensors, vol. 4, Artech House, Boston, Mass, USA, 1997.

[6] S. Muto, O. Suzuki, T. Amano, and M. Morisawa, "A plastic optical fibre sensor for real-time humidity monitoring," Measurement Science and Technology, vol. 14, no. 6, pp. 746-750, 2003.

[7] M. Morisawa, Y. Amemiya, H. Kohzu, C. X. Liang, and S. Muto, "Plastic optical fibre sensor for detecting vapour phase alcohol," Measurement Science and Technology, vol. 12, no. 7, pp. 877$881,2001$. 

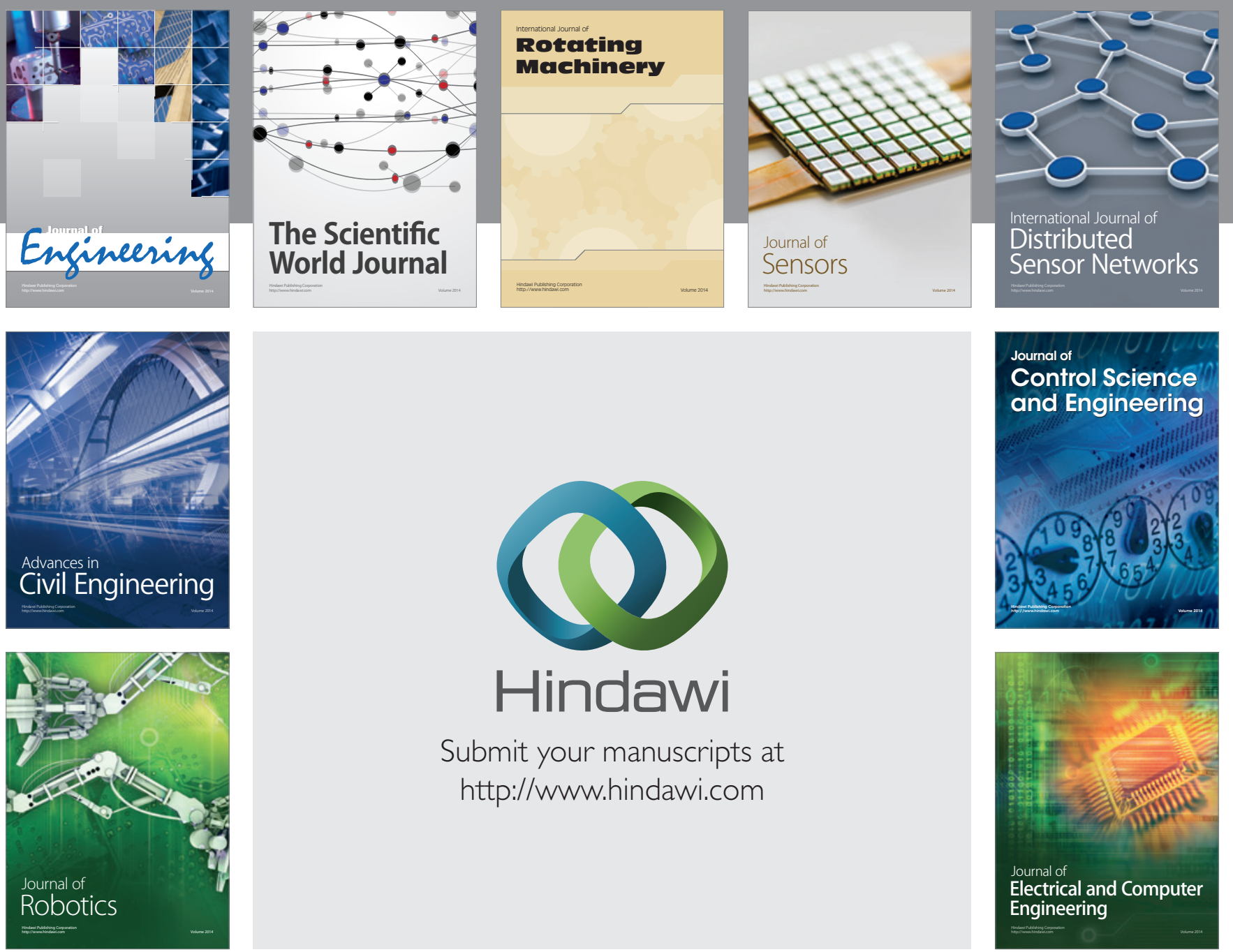

Submit your manuscripts at

http://www.hindawi.com
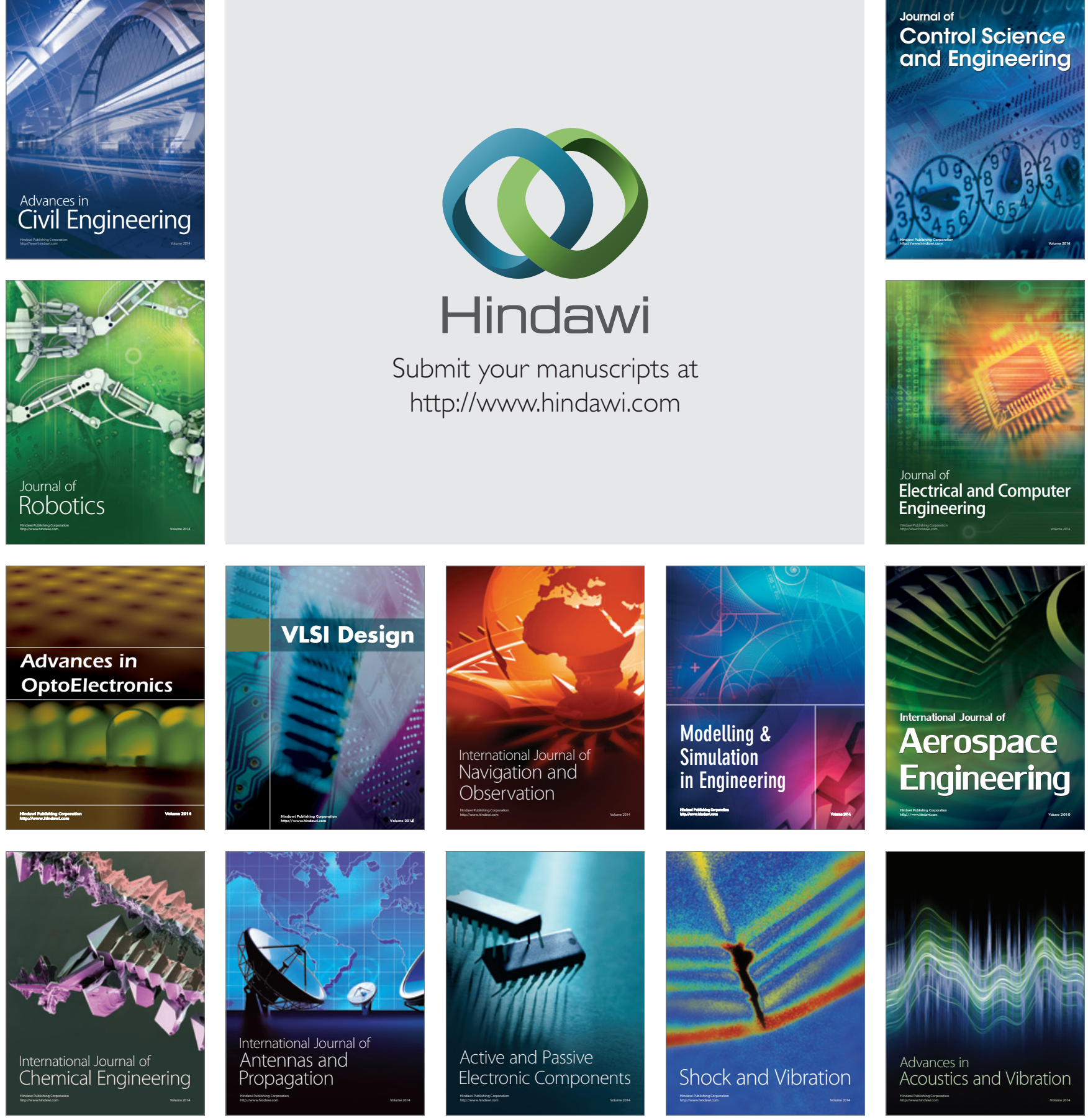